\title{
Movement Behavior of Oil Drops and Demulsification of Oil-in-water(O/W) Emulsion under Direct Current Pulsed Electric Field
}

\author{
Bo-Ping REN ${ }^{a}$ and Yong KANG ${ }^{\text {b,* }}$
}

\author{
School of Chemical Engineering and Technology, Tianjin University, Tianjin 300350, China \\ arenbp2008@163.com, bykang@tju.edu.cn
}

Keywords: O/W emulsion, Demulsification, Direct current pulsed electric field, Coalescence.

\begin{abstract}
The electric demulsification of oil-in-water $(\mathrm{O} / \mathrm{W})$ emulsion was investigated under the application of direct current pulsed electric field (DCPEF). The oil droplet movement behavior was observed and analyzed by CCD camera and image acquisition and analysis system. It was found that DCPEF had an effect on the oil droplet coalescence, which ultimately led to the demulsification of $\mathrm{O} / \mathrm{W}$ emulsion. Through the investigation on the dynamic behavior of oil drops under DCPEF, it was discovered that DCPEF could drive oil droplets to move and form oil droplet chains and oil clusters, which accelerated the demulsification process and achieved the oil-water separation. The results showed that DCPEF had distinct demulsification effect on O/W emulsion. The optimal DCPEF parameters for oil-water separation were obtained that DCPEF voltage of $1000 \mathrm{~V}$, frequency of $25 \mathrm{~Hz}$, and duty cycle of $70 \%$ under the experimental conditions.
\end{abstract}

\section{Introduction}

Emulsions exist widely in industries and human daily life such as crude oil produced water, emulsified drilling fluid, metal machining liquid, and food, pharmaceutical and so on. There are two types of emulsions which are oil-in-water $(\mathrm{O} / \mathrm{W})$ emulsion and water-in-oil (W/O) emulsion [1-3]. In practice, they are easy to be prepared but not easy to be broken especially for $\mathrm{O} / \mathrm{W}$ emulsion. The oily waste water is one kind of $\mathrm{O} / \mathrm{W}$ emulsion. Additionally, a large amount of oily waste water is produced in industry and human life every year. If discharged directly without treatment, it will seriously pollute the environment. At the same time, a lot of oil and water resources also will be wasted [4-6]. The oil phase in oily wastewater mainly exists in the form of emulsified oil[7,8].In order to recover the oil resources and obtain pure water, the demulsification researches are widely carried out, among which the phase separation using electric field draws much interest and endeavor[9,10].

In this work, the movement behavior of oil drops in O/W emulsion was observed by CCD camera. Then the direct current pulsed electric field (DCPEF) was applied to electrically demulsify the O/W emulsion for oil-water separation. The demulsification and separation effect of $\mathrm{O} / \mathrm{W}$ emulsion was evaluated by measuring the clear liquid thickness after the treatment by DCPEF.

\section{Materials and Experimental Setup}

\section{Preparation of $\mathrm{O} / \mathrm{W}$ Emulsion.}

The oil used in this paper was diesel(0\#) purchased from CNPC with the density of $886 \mathrm{~kg} / \mathrm{m}^{3}$ and the viscosity of $31.9 \mathrm{mPa} \cdot \mathrm{s}$ at $293 \mathrm{~K}$. The surfactant SDS (sodium dodecyl sulfate) was analytically pure and purchased from Tianjin Jiang Tian Chemical Co., Ltd. The O/W emulsion was made by pouring the diesel (10 vol.\%) into water solution of SDS (2.5wt.\%) slowly and mixing together vigorously by a high-speed homogenizer (D-8401WZ, Tianjin Huaxing Co., Ltd) at the stirring speed of $3100 \mathrm{rpm}$ for $10 \mathrm{~min}$. After being settled for $24 \mathrm{~h}$, the mix was stirred at $3100 \mathrm{rpm}$ for $10 \mathrm{~min}$ again to make the emulsion more stable. The oil drops diameter maintained in the range of $1-15 \mu$ m measured by the image acquisition and analysis system (BX43 Olympus Microscope, Japan), which has a CCD camera (DP25 U-CMAD3 Olympus, Japan) to record the oil drops with a frame speed of 2000 frames per second. And the emulsion was stirred at $800 \mathrm{rpm}$ during every experiment to keep uniform. 


\section{Experimental Setup and Procedures.}

The schematic diagram of the experimental setup was shown in Fig.1.In the experiments; the high speed CCD camera (f) was firstly fixed at $15 \mathrm{~mm}$ right over the rectangle Perspex cell(c). After that, the electrode sheets (d) as the anode and the cathode were connected with the positive and negative outputs of the pulsed power supply (h) with copper wires, respectively. Then, $10 \mathrm{~mL} \mathrm{O} / \mathrm{W}$ emulsion was fetched and injected to the rectangle Perspex cell from the O/W emulsion tank (a). Then turned on the pulsed power supply and set the values of its voltage, frequency, and duty cycle. The movement process of oil drops under DCPEF was observed and recorded by microscope image acquisition and analysis system (g). After the observation finished, the electrode plates(j) were connected with the positive and negative outputs of the pulsed power supply with copper wires, respectively. Then, $150 \mathrm{~mL} \mathrm{O/W}$ emulsion was poured into the rectangle perspex container(k), where the separation took place. During the separation process, the thickness of the separated clear liquid was recorded by the ruler(i) which was fixed on the outer surface of the rectangle perspex container(k). The separation process of every experiment lasted for $2 \mathrm{~h}$. All the experiments were performed three times and the average values of the results are reported.

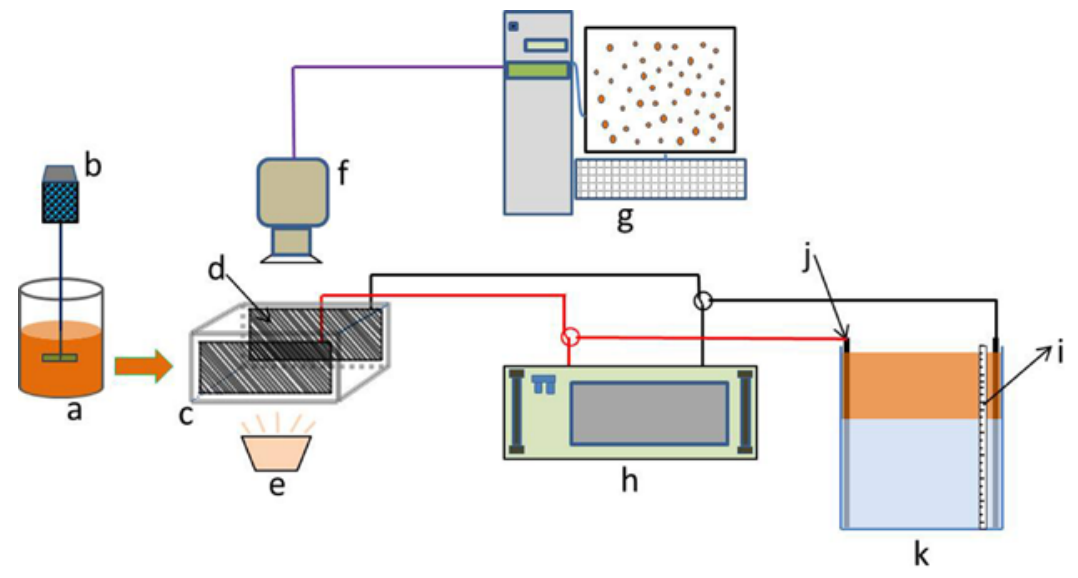

Fig. 1 The schematic diagram of the experimental setup:(a)O/W emulsion tank, (b) stirrer,(c) rectangle perspex cell,(d) a pair of titanium electrode sheets,(e) light source,(f) CCD camera,(g) microscope image acquisition and analysis system,(h) pulsed power supply,(i) ruler,(j) a pair of titanium electrode plates, $(\mathrm{k})$ rectangle perspex container.

\section{Results and Discussion}

\section{Movement Behavior of Oil Drops.}

The dynamic behavior of oil drops in O/W emulsion under DCPEF with voltage of $750 \mathrm{~V}$, frequency of $25 \mathrm{~Hz}$, and duty cycle of 50\% was observed and shown in Fig.2. Before the DCPEF was applied to the $\mathrm{O} / \mathrm{W}$ emulsion, the oil drops was kept stable and uniform as shown in Fig.2a (0s). Once the DCPEF was activated, oil droplets started to move and string together in line and formed oil droplet chains as presented in Fig.b(20s). With DCPEF continuing taking effect, the moving intensity of oil drops increased and there existed oil clusters in the emulsion, which also strung together in line as shown in Fig.2c (85s). When DCPEF lasting for $2 \mathrm{~min}$, the oil clusters merged together and formed a large oil cluster. With DCPEF continuing to function for $12 \mathrm{~min}$, the small oil drops merged and coalesced together to form large oil drops. And the emulsion flowed slowly and became clear viewed from the outside. The oil drops became larger which indicated that the demulsification and the coalescing of oil drops were ongoing continuously as shown in Fig.2f (20min).

It was found that DCPEF had the ability of coalescing oil droplets, which could make oil droplets gather together and the large oil clusters were presented during DCPEF taking effect. During the motion process, oil droplets migrated along the electric field direction and connected with adjacent drops forming oil droplet chains. Then two abreast adjacent oil droplet chains attracted and stuck together with each other forming one oil cluster [11,12]. The formation of oil clusters and large oil 
droplets accelerated the floating speed of oil drops and then accelerated the demulsification process of $\mathrm{O} / \mathrm{W}$ emulsion under DCPEF. Therefore, the oil-water separation of $\mathrm{O} / \mathrm{W}$ emulsion was achieved under the effect of DCPEF.

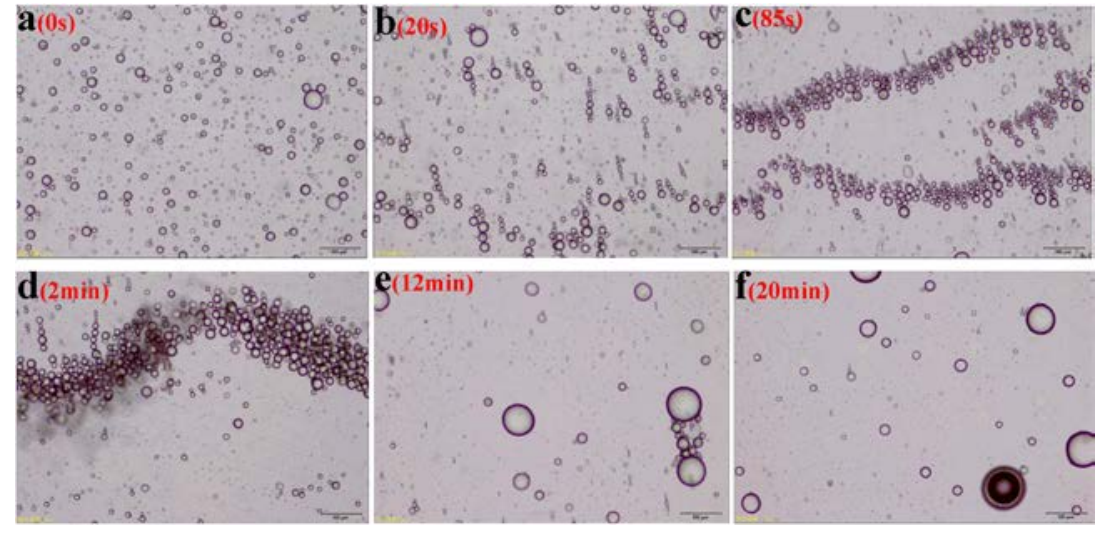

Fig. 2 The dynamic behavior of oil droplets in O/W emulsion under DCPEF with its voltage of $750 \mathrm{~V}$, frequency of $25 \mathrm{~Hz}$, and duty cycle of $50 \%$.

\section{Effects of DCPEF Voltage on Oil-Water Separation Property of O/W Emulsion.}

The separation effect of oil droplets in O/W emulsion under DCPEF voltage was explored as shown in Fig.3. It could be seen that the clear water layer thickness increased with the increasing of DCPEF voltage under the same DCPEF frequency and duty cycle. It was indicated that the DCPEF voltage could accelerate the demulsification process of $\mathrm{O} / \mathrm{W}$ emulsion. This could be explained that when the voltage increasing, the electrostatic force applied on oil drops increased, which drove oil drops approaching and colliding fast. Therefore, the speed of oil drops coalescing and demulsification of $\mathrm{O} / \mathrm{W}$ emulsion increased with the increase of voltage. Therefore, it could be concluded that the optimal DCPEF voltage was $1000 \mathrm{~V}$ under the experimental conditions.

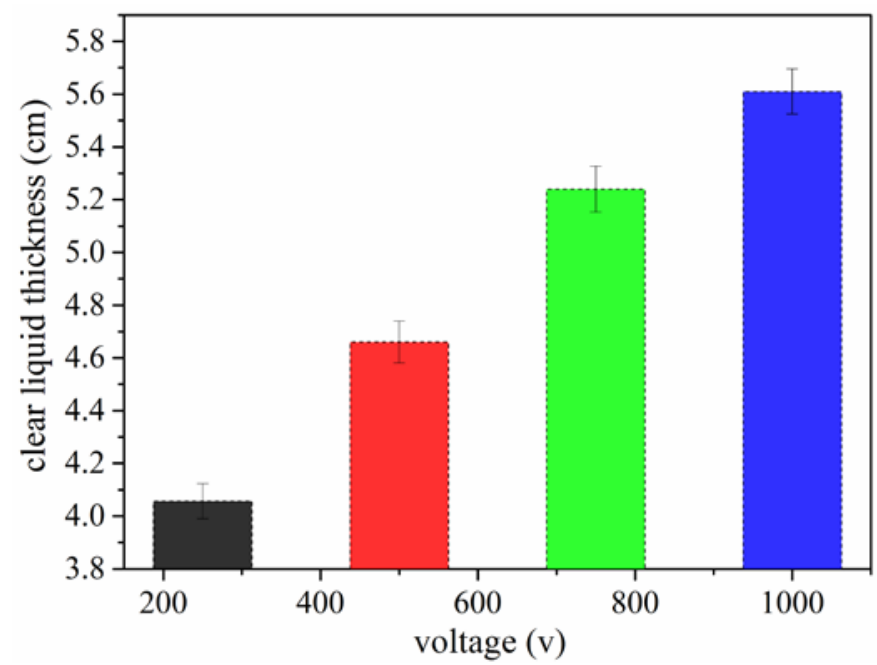

Fig. 3 The clear liquid thickness under different DCPEF voltages with its frequency of $50 \mathrm{~Hz}$ and duty cycle of $50 \%$ after been treated for $2 \mathrm{~h}$.

\section{Effects of DCPEF Frequency on Oil-Water Separation Property of O/W Emulsion.}

The clear liquid thickness under different DCPEF frequencies with its voltage of $1000 \mathrm{~V}$ and duty cycle of 50\% as presented in Fig.4. On the contrary, the clear water layer thickness decreased with the DCPEF frequency increasing, which meant that DCPEF voltage could enhance the separation effect but the DCPEF frequency did the opposite. And the clear liquid thickness reached to the maximum value when the DCPEF frequency declined to the $25 \mathrm{~Hz}$. With the frequency increasing, the duration of one cycle of DCPEF became shorter. The duration of electrostatic force applied on oil drops also 
became shorter, which made the oil drops vibrated in situ and had inadequate time to contact with each other [13]. Then there would be less oil droplet chains and oil clusters formed in O/W emulsion under DCPEF. Therefore, it could be assumed that demulsification effect became worse with the DCPEF frequency increasing and the optimal DCPEF frequency was $25 \mathrm{~Hz}$ under the experimental conditions.

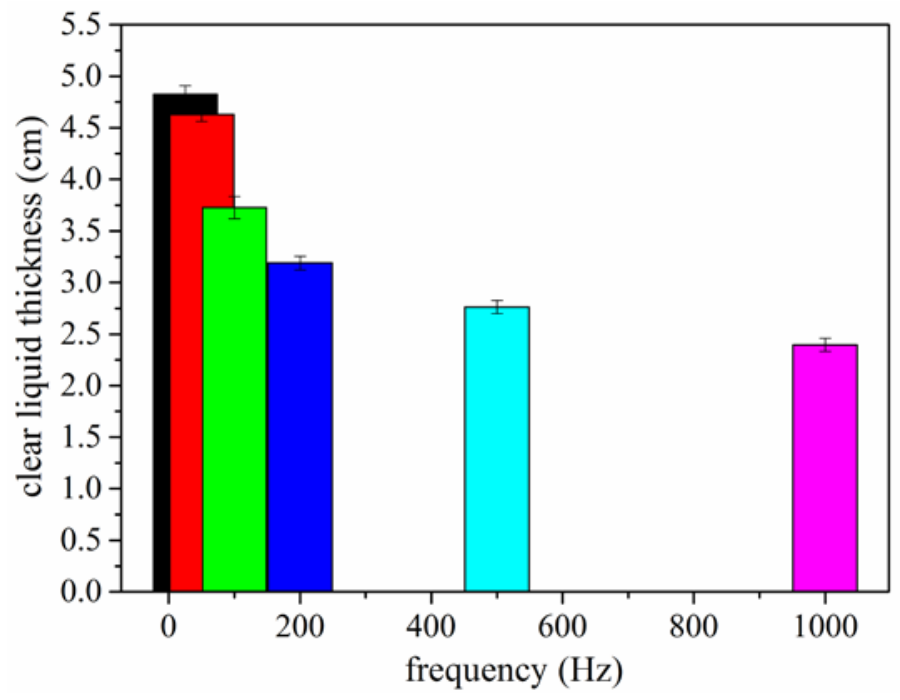

Fig. 4 The clear liquid thickness under different DCPEF frequencies with its voltage of $1000 \mathrm{~V}$ and duty cycle of $50 \%$ after been treated for $2 \mathrm{~h}$.

\section{Effects of Duty Cycle on Oil-Water Separation Property of O/W Emulsion.}

The clear liquid thickness under different DCPEF duty cycles with its voltage of $1000 \mathrm{~V}$ and frequency of $50 \mathrm{~Hz}$ as shown in Fig.5.It could be found that the water layer thickness increased with the duty cycle increasing from $10 \%$ to $70 \%$ and then decreased with the duty cycle continuously increasing to $90 \%$.It was indicated that the separation effect reached to the optimal when the duty cycle was $70 \%$. The duty cycle is the ratio of pulse output time to one cycle time. When the duty cycle increased, the pulse output time increased and the duration of electrostatic force applied on oil drops was longer, which meant that oil drops had sufficient time to form oil drop chains and oil clusters. However, it could be assumed that when DCPEF duty cycle reached to $90 \%$, the duration of electrostatic force increased to a certain extent leading to the collision of oil drops becoming much more violent. Then the oil drops collided and broke into small oil droplets. Thus, the demulsification effect became worse. The optimal demulsification effect of $\mathrm{O} / \mathrm{W}$ emulsion achieved when the DCPEF duty cycle reached to 70\% under the experimental conditions.

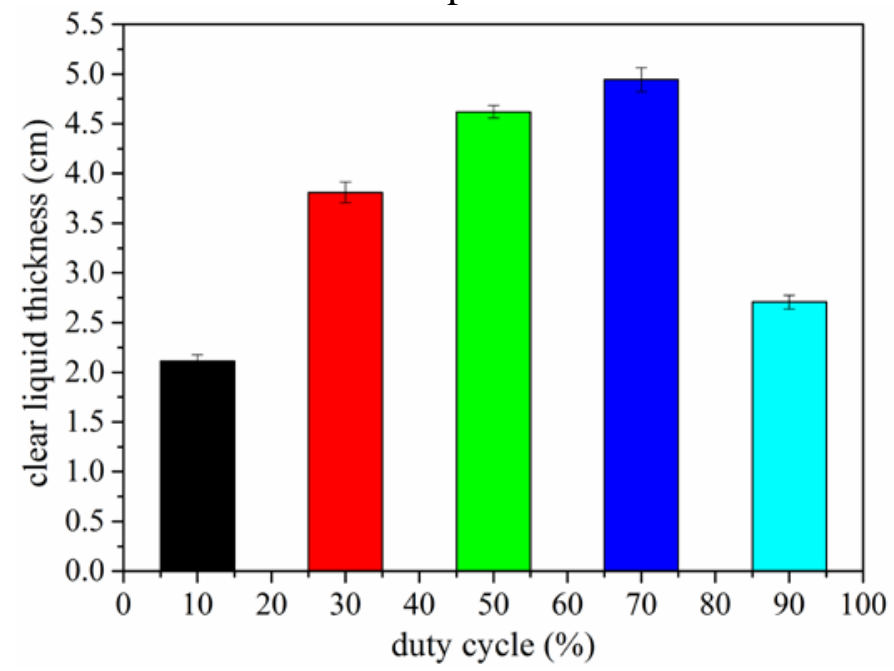

Fig. 5 The clear liquid thickness under different DCPEF duty cycles with its voltage of $1000 \mathrm{~V}$ and frequency of $50 \mathrm{~Hz}$ after been treated for $2 \mathrm{~h}$. 
After that, the $\mathrm{O} / \mathrm{W}$ emulsion was treated under the optimal DCPEF parameters with its voltage of $1000 \mathrm{~V}$, frequency of $25 \mathrm{~Hz}$, and duty cycle of 70\%. The clear liquid after treatment was compared with the raw emulsion as presented in Fig.6. It could be seen from Fig.6b(left) that the clear liquid after treatment was much clearer than the raw $\mathrm{O} / \mathrm{W}$ emulsion and the turbidity of the clear liquid was 14.3 NTU, which was far less than that reported by Ichikawa[6] and Hosseini[8]. The oil droplet distribution in treated clear liquid was presented in Fig.6b(right), from which there were very few oil drops remaining in the clear liquid.

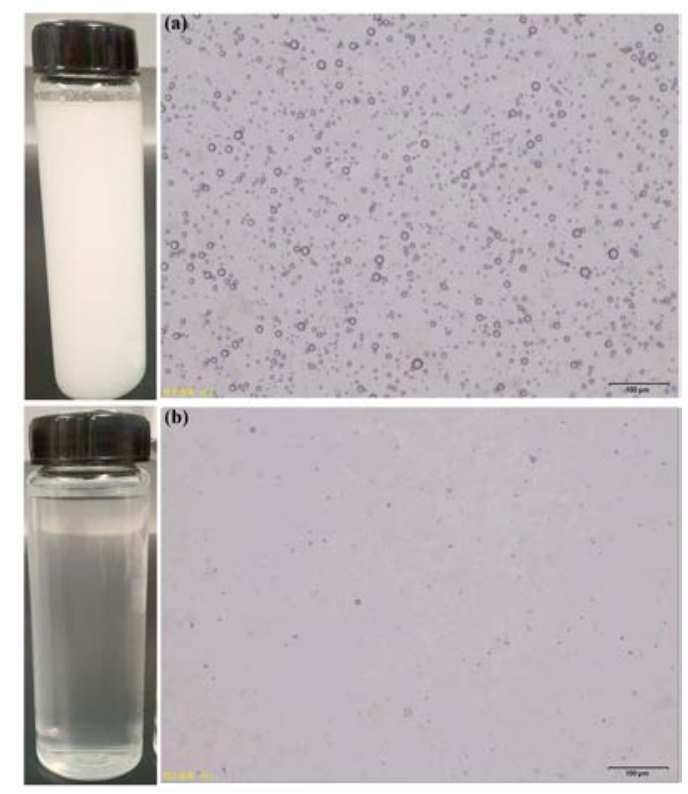

Fig. 6 (a)The microscopic photo of O/W emulsion before processing(right) and the raw emulsion(left).(b) The microscopic photo of $\mathrm{O} / \mathrm{W}$ emulsion after processing(right) and the clear liquid(left).

\section{Conclusions}

The DCPEF had evident demulsification effect on O/W emulsion. And it could be concluded that DCPEF can drive the oil droplets to form oil droplet chains and oil clusters and the demulsification took place during DCPEF applied. The increase of DCPEF voltage can promote oil droplets to coalesce and the demulsification effect. The separation effect was enhanced by the DCPEF voltage and the DCPEF frequency did the opposite. The optimal duty cycle was $70 \%$ for the best separation. Therefore, The optimal DCPEF parameters for the demulsification of $\mathrm{O} / \mathrm{W}$ emulsion were obtained that DCPEF voltage of $1000 \mathrm{~V}$, frequency of $25 \mathrm{~Hz}$, and duty cycle of $70 \%$ under the experimental conditions.

\section{Acknowledgement}

This research did not receive any specific grant from funding agencies in the public, commercial, or not-for-profit sectors.

\section{References}

[1] S. Mhatre, V. Vivacqua, M. Ghadiri, A.M. Abdullah, M.J. Al-Marri, A. Hassanpour, B. Hewakandamby, B. Azzopardi, B. Kermani, Electrostatic phase separation: A review, Chemical Engineering Research \& Design, 96 (2015) 177-195.

[2] S. Less, R. Vilagines, The electrocoalescers' technology: Advances, strengths and limitations for crude oil separation, Journal of Petroleum Science \& Engineering, 81 (2012) 57-63. 
[3] D. Yang, M. Xu, L. He, X. Luo, Y. Lü, H. Yan, C. Tian, The influence and optimisation of electrical parameters for enhanced coalescence under pulsed DC electric field in a cylindrical electrostatic coalescer, Chem. Eng. Sci., 138 (2015) 71-85.

[4] H. Yan, L. He, X. Luo, J. Wang, The study of deformation characteristics of polymer droplet under electric field, Colloid \& Polymer Science, 293 (2015) 2045-2052.

[5] G. Berg, L.E. Lundgaard, N. Abi-Chebel, Electrically stressed water drops in oil, Chemical Engineering \& Processing Process Intensification, 49 (2010) 1229-1240.

[6] T. Ichikawa, K. Itoh, S. Yamamoto, M. Sumita, Rapid demulsification of dense oil-in-water emulsion by low external electric field: I. Experimental evidence, Colloids \& Surfaces A Physicochemical \& Engineering Aspects, 242 (2004) 21-26.

[7] T. Ichikawa, Electrical demulsification of oil-in-water emulsion, Colloids \& Surfaces A Physicochemical \& Engineering Aspects, 302 (2007) 581-586.

[8] M. Hosseini, M.H. Shahavi, A. Yakhkeshi, AC \& DC-currents for separation of nano-particles by external electric field, Asian Journal of Chemistry, 24 (2012) 181-184.

[9] M. Chiesa, S. Ingebrigtsen, J.A. Melheim, P.V. Hemmingsen, E.B. Hansen, Ø. Hestad, Investigation of the role of viscosity on electrocoalescence of water droplets in oil, Separation \& Purification Technology, 50 (2006) 267-277.

[10] V. Vivacqua, S. Mhatre, M. Ghadiri, A.M. Abdullah, A. Hassanpour, M.J. Al-Marri, B. Azzopardi, B. Hewakandamby, B. Kermani, Electrocoalescence of water drop trains in oil under constant and pulsatile electric fields, Chemical Engineering Research \& Design, 104 (2015) 658-668.

[11] J.S. Eow, M. Ghadiri, A.O. Sharif, T.J. Williams, Electrostatic enhancement of coalescence of water droplets in oil: a review of the current understanding, Chemical Engineering Journal, 84 (2002) 173-192.

[12] M. Mousavichoubeh, M. Ghadiri, M. Shariaty-Niassar, Electro-coalescence of an aqueous droplet at an oil-water interface, Chemical Engineering \& Processing Process Intensification, 50 (2011) 338-344.

[13] J.S. Eow, M. Ghadiri, Motion, deformation and break-up of aqueous drops in oils under high electric field strengths, Chemical Engineering \& Processing Process Intensification, 42 (2003) 259-272. 